\title{
Abdominal Actinomycosis: A Case Report
}

\author{
Seyed Alireza Mousavi ${ }^{1}$, Seyed Masoud Miratashiyazdi ${ }^{1}$, Saeed Kargar $^{2}$, Seyed Hossein Shahcheraghi ${ }^{1,3}$ \\ ${ }^{1}$ Infectious Diseases Research Center, Shahid Sadoughi Hospital, Shahid Sadoughi University of Medical Sciences, Yazd, \\ Iran \\ ${ }^{2}$ Surgery Specialist, Shahid Sadoughi Hospital, Shahid Sadoughi University of Medical Sciences, Yazd, Iran \\ ${ }^{3}$ Department of Modern Sciences \& Technologies, School of Medicine, Mashhad University of Medical Sciences, Mashhad, \\ Iran
}

\begin{abstract}
Actinomycosis is created by a gram-positive organism that does not exist uninhibitedly in nature, but rather ordinary is colonized in the parts including gastrointestinal and genital tract in female and oropharynx. It is well known for its ability to develop into aggressive masses even without fistulas. Here, we introduce a case with abdominal actinomycosis that is one of the particular clinical types of infection. J Microbiol Infect Dis 2020; 10(1):56-58.
\end{abstract}

Keywords: Actinomycosis, Abdominal, Gastrointestinal

\section{INTRODUCTION}

Actinomycosis is unusual, painless and chronic infection caused by the bacteria related to Actinomyces species [1]. It is created by a grampositive organism that does not exist uninhibitedly in nature, but rather ordinary is colonized in the parts including gastrointestinal and genital tract in female and oropharynx [1]. Human type of disease is commonly frequent in the cervicofacial area (55\%) and following thoracic (25\%) [2]. The prevalence has decreased overall because of increased oral hygiene and the advancement of antibiotics [2]. Actinomyces is well known for its ability to develop into aggressive masses even without fistulas when has destroyed tissue integrity and is often underdiagnosed due to its similarity with characteristics of other infections (e.g. tuberculosis and nocardiosis) [3].

\section{METHODS}

A 52-year-old woman, HIV negative and no history of treatment with antibiotic and fungal drugs, presented with a one-year history of abdominal dull pain in Right Lower Quadrant (RLQ) which walking aggravates and lying alleviates it. The pain intensified from 3 months ago and accompanied by nausea and vomiting from a month ago a red palpable swelling mass emerged in RLQ which also had a bloody discharge (Figure 1).

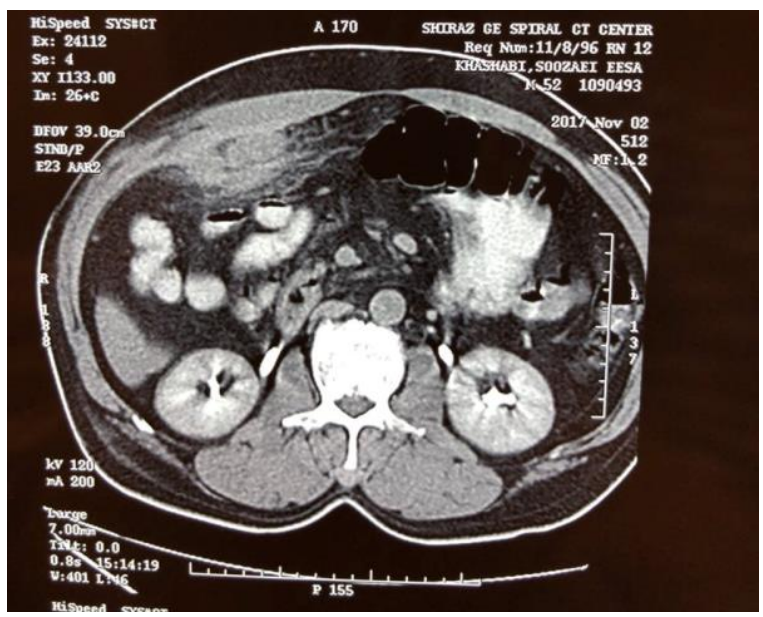

Figure 1. Computed tomography of abdominopelvic showing a $3 \times 4 \mathrm{~cm}$ mass near abdominal wall.

The patient had Diabetes Mellitus from 1 year ago and had a history of appendectomy complicated with intestinal fistula about 8 years

Correspondence: Dr. Seyed Hossein Shahcheraghi, Department of Modern Sciences \& Technologies, School of Medicine, Mashhad University of Medical Sciences, Mashhad \& Infectious Diseases Research Center, Shahid Sadoughi Hospital, Shahid Sadoughi University of Medical Sciences, Yazd, Iran E-mail: shahcheraghih@gmail.com

Received: 11 June 2019 Accepted: 28 November 2019

Copyright (C JMID / Journal of Microbiology and Infectious Diseases 2020, All rights reserved 
ago. She also had hypertension, hyperlipidemia, hemorrhoid, and a history of curettage due to thickening of endometriosis in 15 months ago. In other assessments CXR was normal. The patient vital signs were in the normal range except for hypertension $(140 / 80 \mathrm{mmHg}$ ) and initial laboratory testing revealed hyperglycemia and normal leukocyte count (BS: $325 \mathrm{mg} / \mathrm{dl}$, WBC 5100, Hb: $13.5 \mathrm{mg} / \mathrm{dl}$, Plt: 329,000). The patient went under laparotomy due to probable fistula. During the operation an abscess which had a fistula to skin was determined cecumectomy was carried out and cutaneous fistula sealed. Histopathological examination of the abscess discharges showed actinomycosis sensitive to penicillin (according to disk diffusion test). As soon as the pathology report became available, intramuscular penicillin treatment was initiated followed by oral penicillin. She also received regular insulin due to her hyperglycemia and discharged with oral penicillin to complete 12 months course.

\section{DISCUSSION}

Actinomyces is usually considered a saprophyte in the gastrointestinal tract, and genital tract, including mechanical wounds, operations, intrauterine contraceptive device placement and tooth extraction can totally destroy the mucosal barrier structure, which makes it easier for the pathogen to penetrate deeper into the tissue [4]. Our case predisposing variables are the history of appendectomy and currtage, although $50 \%$ of patients have no predisposing factor [5]. Abdominopelvic actinomycosis is one of the particular clinical types of infected sites. It is infrequent, but it results in tissue granulation, abscess and dense fibrosis, leading to a hard pelvic mass compressing ureter, the urinary bladder and rectum [6]. The main abdominopelvic actinomycosis usually imitates an intra- abdominal tumor or malignancy because of its capacity to establish a mass infiltrated while postoperative actinomycosis occurs more frequently as abscess [7]. Our patient was considered with a skin fistula with peritoneal mass, while it might be a single abscess of the liver, mimics an inflammatory bowel disease or pelvic abscess $[4,8]$. Similar to our case, other cases of actinomycosis commonly do not cause signs or symptoms of the characteristic disease and generally manifests itself as a slow- growing mass, which can be related to vomiting, nausea, altered bowel habit changes and pain [6]; causes preoperative diagnosis mistake and is found in only less than $10 \%$ of cases and histopathological examination in other cases is the most used diagnostic methods after an operation [6]. The standard medical therapy for uncomplicated cases is long- term treatment with penicillin [9] which was also helpful in our patient.

\section{Conclusion}

Late diagnosis of actinomycosis infection continues to be difficult, postponing the proper treatment and enhancing the length of infection period. It should be included and considered in the differential diagnosis in patients with abdominal masses with malignant or inflammatory characteristics due to its nonspecific symptoms.

\section{ACKNOWLEDGMENTS}

The authors would like to thank staff of Infectious Diseases Research Center of Yazd Shahid Sadoughi University of Medical Sciences for their kind assistance in performing this study.

Declaration of Conflicting Interests: The authors declare that they have no conflict of interest.

Financial Disclosure: No financial support was received.

\section{REFERENCES}

1. Sampaio C, Pina S, Frade S, Moniz L. Surgical treatment for unusual presentations of abdominal actinomycosis: two case reports. Pan Afr Med J 2017;28:133.

2. Heo SH, Shin SS, Kim JW, et al. Imaging of actinomycosis in various organs: a comprehensive review. Radiographics. 2014;34:19-33.

3. Paulo CO, Jordão S, Correia-Pinto J, Ferreira F, Neves I. Actinomycosis, a lurking threat: a report of 11 cases and literature review. Revista da Sociedade Brasileira de Medicina Tropical. 2018; 51:7-13

4. Hayashi M, Asakuma M, Tsunemi S, et al. Surgical treatment for abdominal actinomycosis: A report of two cases. World J Gastrointestinal Surg $2010 ; 2: 405$.

5. Heidt J, Jansen C, Leyten E. An abdominal mass: not a 'clear cut' case! Netherlands J Med 2010; 68:319-21. 
6. Yang SS, Im YC. Severe abdominopelvic actinomycosis with colon perforation and hepatic involvement mimicking advanced sigmoid colon cancer with hepatic metastasis: a case study. BMC Surgery 2018; 18:51.

7. Fazili T, Blair D, Riddell S, Kiska D, Nagra S. Actinomyces meyeri infection: case report and review of the literature. J Infection 2012; 65:357361.

8. Markey CM, Vestal LE. Actinomyces meyeri: A Rare Cause of Postsurgical Pelvic Actinomycosis. Case Reports in Obstetrics and Gynecology. 2018; 2018.

9. Valour F, Sénéchal A, Dupieux $C$, et al. Actinomycosis: etiology, clinical features, diagnosis, treatment, and management. Infect Drug Resist 2014;7:183. 